\title{
Stimulus duration effects on vibrotactile magnitude estimation for the tongue and hand
}

\author{
DONALD FUCCI \\ Ohio University, Athens, Ohio \\ LINDA PETROSINO \\ University of North Carolina, Chapel Hill, North Carolina \\ DANIEL HARRIS \\ Brown Schools, Ranch Treatment Center, Austin, Texas
}

and

ELISE MCMATH

Ohio University, Athens, Ohio

\begin{abstract}
The present study was designed to investigate the effect of stimulus duration on vibrotactile sensation magnitude for the lingual dorsal surface and the thenar eminence of the right hand through use of the psychophysical scaling method of magnitude estimation. Magnitude estimation values for the tongue and hand were derived from 20 normal young adults. Measurements were made at a frequency of $250 \mathrm{~Hz}$ with stimulus durations of 100,500 , and $1,000 \mathrm{msec}$. Results showed the upper power function exponents to be steeper at all three stimulus durations for the lingual dorsal surface than for the thenar eminence, and the exponents for both the tongue and hand were smaller for $1,000 \mathrm{msec}$ than for $100 \mathrm{msec}$. Pearson product moment correlations were performed on the subject magnitude estimation responses for the three stimulus durations. The durational correlations were performed independently for both test structures. Correlation coefficients for both test structures at all three stimulus durations tended to increase as stimulus intensity was increased. The correlation coefficients did not reflect changes in the relationship between individual subject responses as a result of stimulus durational changes at any of the sensation levels tested. Results are compared to previous research and discussed in terms of internal scaling mechanisms and learning effect.
\end{abstract}

Von Békésy (1959b) investigated the effect that stimulus duration has on hearing and skin sensations. He suggested that for vibratory stimulation of the skin, there is an initial increase in sensation magnitude during the first second of stimulation, followed by a gradual decline thereafter. Verrillo (1965) explored the effect of stimulus duration on vibrotactile thresholds of the hand and determined that vibrotactile thresholds decreased with increasing duration up to about $600 \mathrm{msec}$. Verrillo (1968) did not find a similar stimulus duration effect for vibrotactile thresholds of the tongue.

Berglund, Berglund, and Ekman (1967), using a direct scaling method, studied the effect of stimulus duration on the subjective magnitude of suprathreshold vibrotactile stimuli delivered to the finger. They found that subjective intensity of a $250-\mathrm{Hz}$ signal was a logarithmic function of stimulus duration up to $1 \mathrm{sec}$. These researchers reported power function exponents that decreased from .70 for a duration of $30 \mathrm{msec}$ to .40 for durations of $220 \mathrm{msec}$ or longer. Gescheider (1976) reported

Please address correspondence to D. Fucci, School of Hearing and Speech Sciences, Ohio University, Athens, OH 45701. suprathreshold magnitude estimation exponent values for the hand of .43 for durations of 200 and $50 \mathrm{msec}$, and .40 for a duration of $600 \mathrm{msec}$. Verrillo and Smith (1976) used the method of magnitude estimation to study stimulus duration effect on the thenar eminence of the right hand. They reported power function exponents of .50 for $10 \mathrm{msec}, .49$ for $80 \mathrm{msec}$, and .47 for $600 \mathrm{msec}$. Although the methodologies varied considerably among these investigations, in each, a stimulus duration effect was noted whereby the suprathreshold power function exponent decreased with an increase in stimulus duration.

The test sites for vibrotactile stimulation used in the research described above were selected from various locations on the hand. To date, the possible effects of stimulus duration at suprathreshold levels has not been investigated for other body locations. The purpose of the present study was to extend the research on vibrotactile sensation magnitude by investigating stimulus duration effect on the dorsal surface of the tongue and the thenar eminence of the right hand through use of the psychophysical scaling method of magnitude estimation. A comparison of sensation magnitude scaling data between these two test sites may provide additional information concern- 
ing temporal summation at body locations which vary in overall neurophysiological characteristics (Bosma, 1967).

\section{METHOD}

\section{Subjects}

Twenty subjects, 10 men and 10 women, aged 18-27 years (mean $=20.45$ years), were selected randomly from an introductory course in speech and hearing sciences at Ohio University. All subjects had normal speech and hearing and reported no known sensory and/or motor impairments. None of the subjects were experienced in vibrotactile testing.

\begin{abstract}
Apparatus
The vibrotactile instrumentation consisted of a stimulus unit and a measurement unit. The stimulus unit was composed of a sine-wave generator, an experimenter-controlled variable attenuator, a rise/fall gate, two universal timers, an audio amplifier, a power amplifier, and an electromagnetic minivibrator with a probe-contactor extension. The pulsed vibratory signal generated had a frequency of $250 \mathrm{~Hz}$, with a rise-decay time of $5 \mathrm{msec}$. Three signal durations of 100,500 , and $1,000 \mathrm{msec}$ were selected, with a constant interstimulus interval of $2 \mathrm{sec}$. The measurement unit consisted of an accelerometer, a cathode follower, a microphone amplifier, and a voltmeter. A narrow band generator was used to present auditory masking at 70-dB HTL to the subjects in order to prevent them from hearing the vibratory stimulus. A detailed description of the vibrotactile equipment and procedures can be found in a review by Fucci, Petrosino, Wallace, and Small (1982).
\end{abstract}

\section{Procedure}

All 20 subjects participated in two test sessions with an interval of 1 week between sessions. A split-half design was used in which half of the subjects were tested on the lingual dorsal surface during the first test session and the thenar eminence of the right hand during the second test session. The remaining subjects were tested on the thenar eminence of the right hand during the first test session and the lingual dorsal surface during the second test session. During each session, three signal durations of 100,500 , and $1,000 \mathrm{msec}$ were randomly presented in a two-trial series to the structure being tested. In each session, the subject was seated in an adjustable chair and positioned so that the test structure was placed in contact with the lower side of a rigidly mounted plastic disk. A hole in the center of the disk provided access for the contactor extension of the vibrator to the test structure. The contactor had a surface area of $.128 \mathrm{~cm}^{2}$, and there was a 1-mm gap between the contactor and the plastic disk. Each subject's vibrotactile threshold was determined by the ascending method of limits. The accepted threshold was the mean of three successive readings within a 5-mV range. The psychophysical method of magnitude estimation was used to obtain a subjective magnitude function for the tongue and hand (Stevens, 1955). The subjects were required to assign numbers to a randomly presented series of eight stimulus intensities ranging from 6 to $40 \mathrm{~dB}$ SL. To minimize possible biases in the results, no reference standard was provided during the task (Hellman \& Zwislocki, 1963). Each subject was instructed to assign a number to each stimulus intensity such that his or her impression of how large the number was matched his or her impression of how intense the vibration was. Whole numbers, decimals, and fractions were designated as permissible selections (Zwislocki \& Goodman, 1980). The geometric means of the two trials of the eight stimulus intensities were taken as the magnitude estimation responses.

\section{RESULTS AND DISCUSSION}

The mean magnitude estimation functions produced by the 20 subjects for the three stimulus durations presented to the thenar eminence of the right hand are shown in Figure 1. For the hand, the upper slope values for the durations of 100,500 , and $1,000 \mathrm{msec}$ were $.46, .48$, and .42 , respectively. The mean magnitude estimation func-

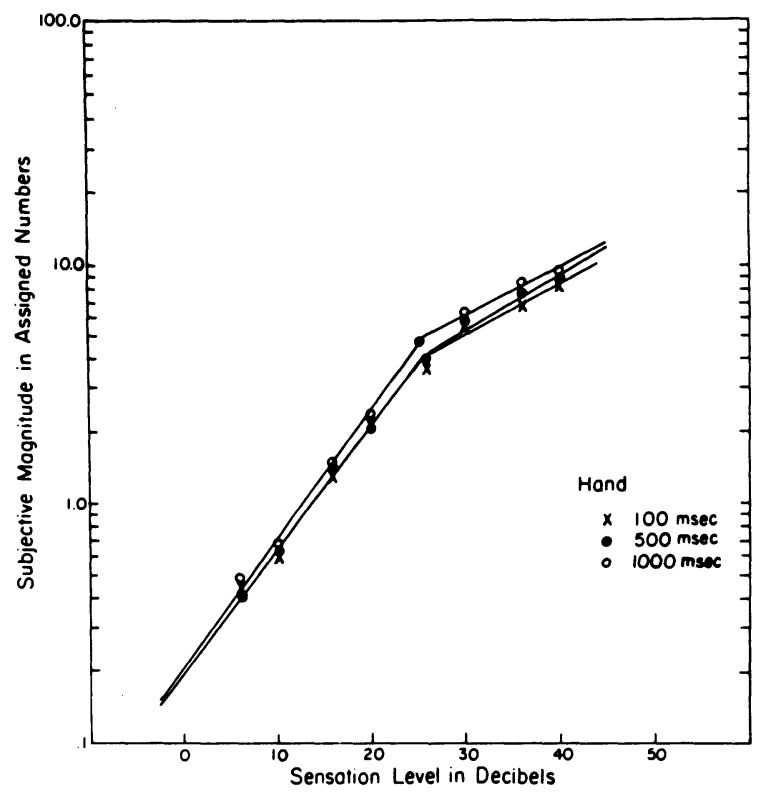

Figure 1. Magnitude estimation functions for three stimulus durations performed on the thenar eminence of the right hand.

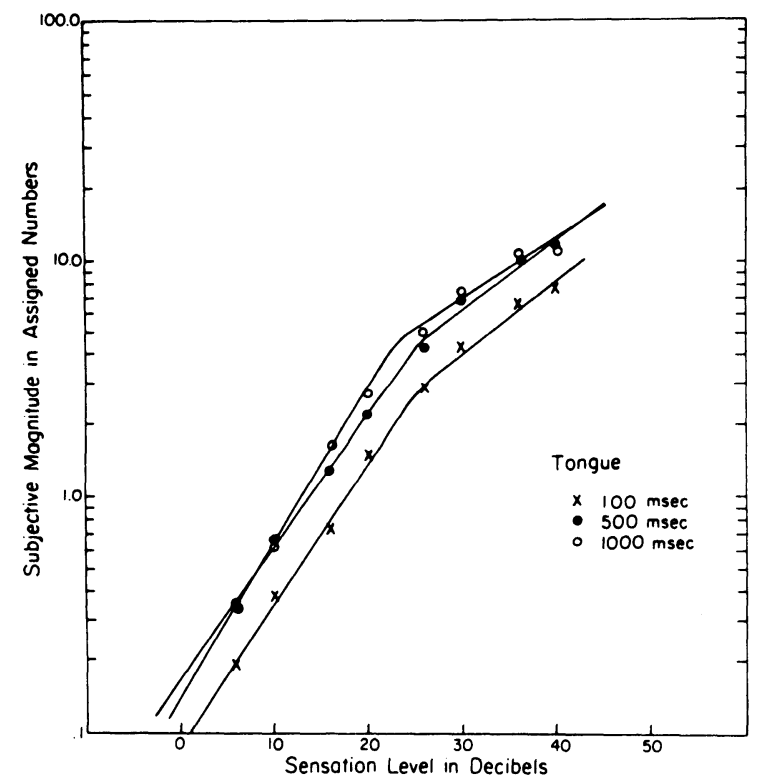

Figure 2. Magnitude estimation functions for three stimulus durations performed on the dorsal surface of the tongue.

tions produced by the 20 subjects for the three stimulus durations presented to the lingual dorsal surface are shown in Figure 2. For the tongue, the upper slope values for the durations of 100,500 , and $1,000 \mathrm{msec}$ were $.66, .62$, and .50 , respectively. The lower slope values for the magnitude estimation functions produced by the 20 subjects for the three stimulus durations for both test structures approached unity. These lower slope findings are in agreement with Zwislocki's theory of temporal summation (Zwislocki, 1960, 1965; Hellman \& Zwislocki, 1961), 
which states that sensation magnitude is directly proportional to stimulus energy near threshold of sensitivity.

The upper slope values for the three stimulus durations for the thenar eminence were similar for 100 and $500 \mathrm{msec}$, and showed a slight decrease for $1,000 \mathrm{msec}$. These findings are compatible with those reported by other researchers (Berglund, Berglund, \& Ekman, 1967; Gescheider, 1976; Verrillo \& Smith, 1976). The upper slope values for the thenar eminence were lower than those for the lingual dorsal surface at all three stimulus durations. This finding is in agreement with those of von Békésy (1957, 1959a), Stevens (1959), and Verrillo and Chamberlain (1972). These researchers noted that body locations of lower neural density yield steeper slopes of subjective magnitude than those of higher neural density. The upper slope values for the lingual dorsal surface, like those for the thenar eminence, showed an overall decrease with an increase in stimulus duration.

In order to compare the relationship between individual responses at each of the eight stimulus intensity levels, Pearson product-moment correlations were performed on the subject magnitude estimation responses for the three stimulus durations. These durational correlations were performed independently for both test structures. These findings are shown in Table 1 . It can be seen that correlations for both test structures tended to increase as stimulus intensity level was increased. This trend was evident for the correlations between all three stimulus durations.

The magnitude estimation power function exponents obtained in this study suggest that the subject responses for the lingual dorsum and thenar eminence were similar over the three suprathreshold vibrotactile stimulus durations. In both instances, the upper slope values were smaller for $1,000 \mathrm{msec}$ than they were for $100 \mathrm{msec}$. This finding agrees with the results obtained by Verrillo and Smith (1976), who concluded that subjective magnitude functions are directly proportional to energy near threshold but become compressed at higher intensity levels. In the present study, additional amounts of stimulus intensity did not elicit proportional subjective responses at the higher sensation levels, and this compression of magnitude functions became slightly more noticeable with stimulus duration increases (Figures 1 and 2; Verrillo \& Smith, 1976).

The Pearson product-moment correlations obtained in the current investigation indicated that the relationships be-

\section{Table 1}

Summary Table of Pearson Product-Moment Correlations for Subject Magnitude Estimation Responses

\begin{tabular}{|c|c|c|c|c|c|c|}
\hline \multirow{2}{*}{$\begin{array}{l}\text { Sensation } \\
\text { Level in } \mathrm{dB}\end{array}$} & \multicolumn{2}{|c|}{$100 / 500 \mathrm{msec}$} & \multicolumn{2}{|c|}{$500 / 1,000 \mathrm{msec}$} & \multicolumn{2}{|c|}{$100 / 1,000 \mathrm{msec}$} \\
\hline & Hand & Tongue & Hand & Tongue & Hand & Tongue \\
\hline 6 & .56 & .64 & .57 & .46 & .39 & .71 \\
\hline 10 & .55 & .72 & .55 & .77 & .40 & .76 \\
\hline 16 & .52 & .50 & .70 & .72 & .37 & .48 \\
\hline 20 & .67 & .71 & .62 & .66 & .46 & .78 \\
\hline 26 & .54 & .74 & .86 & .81 & .60 & .70 \\
\hline 30 & .49 & .89 & .78 & .83 & .83 & .86 \\
\hline 36 & .69 & .73 & .90 & .68 & .88 & .73 \\
\hline 40 & .82 & .80 & .97 & .80 & .86 & .88 \\
\hline
\end{tabular}

tween individual subject magnitude estimation responses showed little change over the three stimulus durations at any of the sensation levels tested (Table 1). The correlations were generally higher for the higher sensation levels than for the lower ones, and this trend was consistent for all three stimulus durations. This pattern of correlations suggests that a wider range of numerical responses was used between subjects at lower sensation levels. At higher sensation levels, the range of numeric responses was narrowed between subjects. It appears from the data that the subjects in the present study used similar scaling behaviors for all three durations. This consistent behavior might be reflective of an internal absolute scaling mechanism that is established early in life and applied to all types of numerical scaling behavior (Zwislocki \& Goodman, 1980).

The nature of the design for the present study was such that all three duration sequences $(100,500,1,000 \mathrm{msec})$ were presented in the same experimental session for both the tongue and the hand. Because of this arrangement, it is possible that the consistent behavior across durations was the result of a learning effect that took place during exposure to the first sequence of eight stimulus intensity levels. This learning effect could have influenced the subjects' scaling behavior on the subsequent stimulus duration presentations. R. Teghtsoonian (1973) and Teghtsoonian and Teghtsoonian (1983) reported that a learning effect can take place in psychophysical scaling experimentation. For scaling responses to be independent of this learning effect, these researchers suggest that a minimum of $24 \mathrm{~h}$ should be required between scaling sessions.

In conclusion, the results of this study suggest that the effects of suprathreshold vibrotactile stimulus duration on magnitude estimation functions for the tongue and hand are similar. At lower sensation levels, subjective magnitude appears to be approximately proportional to stimulus intensity for both structures. At the higher sensation levels, compression of magnitude functions for the lingual dorsum appeared to increase with increases in stimulus duration. This pattern of upper slope compression agrees with data previously obtained for vibrotactile stimulation of the hand and suggests that the effects of temporal summation on sensation magnitude may be similar for the tongue and the hand.

\section{REFERENCES}

Berglund, B., Berglund, U., \& Ekman, G. (1967). Temporal integration of vibrotactile stimulation. Perceptual \& Motor Skills, 25, 549-560.

Bosma, J. F. (1967). Symposium on oral sensation and perception. Springfield, IL: Thomas.

Fucci, D., Petrosino, L., Wallace, D., \& Small, L. (1982). Modification of instrumentation for research on lingual vibrotactile sensitivity: Elimination of the tongue clamping procedure. Review of Scientific Instruments, 53, 1294-1296.

GESCHEIDER, G. A. (1976). Evidence in support of the duplex theory of mechanoreception. Sensory Processes, 1, 68-76.

Hellman, R. P., \& Zwislocki, J. (1961). Some factors affecting the estimation of loudness. Journal of the Acoustical Society of America, 33, 687-694.

Hellman, R. P., \& Zwislocki, J. (1963). Monaural loudness func- 
tion at $1000 \mathrm{cps}$ and interaural summation. Journal of the Acoustical Society of America, 35, 856-865.

STEVENS, S. S. (1955). The measurement of loudness. Journal of the Acoustical Society of America, 27, 815-820.

Stevens, S. S. (1959). Tactile vibration: Dynamics of sensory intensity. Journal of Experimental Psychology, 57, 210-218.

Teghtsoonian, R. (1973). Range effects in psychophysical scaling and a revision of Stevens' Law. American Journal of Psychology, 86, 3-27.

Teghtsoonian, M., \& Teghtsoonian, R. (1983). Consistency of individual exponents in cross-modality matching. Perception \& Psychophysics, 33, 203-214.

VERRILLO, R. T. (1965). Temporal summation in vibrotactile sensitivity. Journal of the Acoustical Society of America, 37, 843-846.

VerRillo, R. T. (1968). A duplex mechanism of mechanoreception. In D. R. Kenshalo (Ed.), The skin senses. Springfield, IL: Thomas.

Verrillo, R. T., \& Chamberlain, S. C. (1972). The effect of neural density and contactor surround on vibrotactile sensation magnitude. Perception \& Psychophysics, 11, 117-120.

Verrillo, R. T., \& SMith, R. L. (1976). Effect of stimulus duration on vibrotactile sensation magnitude. Bulletin of the Psychonomic Society, 8, 112-114.

vON BÉKÉSY, G. (1957). Neural volleys and the similarity between some sensations produced by tones and by skin vibrations. Journal of the Acoustical Society of America, 29, 1059-1069.

voN BÉKÉSY, G. (1959a). Neural funneling along the skin and between the inner and outer hair cells of the cochlea. Journal of the Acoustical Society of America, 31, 1236-1249.

VON BÉKÉSY, G. (1959b). Similarities between hearing and skin sensations. Psychological Review, 66, 1-22.

ZwISLOCKI, J. (1960). Theory of temporal auditory summation. Journal of the Acoustical Society of America, 32, 1046-1060.

ZwISLOCKI, J. (1965). Analysis of some auditory characteristics. In R. D. Luce, R. R. Bush, \& E. Galanter (Eds.), Handbook of mathematical psychology (Vol. 3). New York: Wiley.

ZwislockI, J., \& Goodman, D. (1980). Absolute scaling of sensory magnitudes: A validation. Perception \& Psychophysics, 28, 28-38.

(Manuscript received for publication December 16, 1985.) 